\title{
Meibomian Gland Morphology is a Sensitive Early Indicator of Meibomian Gland Dysfunction
}

Short title: Meibomian Gland Morphology and Meibomian Gland Dysfunction

Muhammed Yasin Adil ${ }^{1,2}$, Jiaxin Xiao ${ }^{1,2}$, Jonatan Olafsson ${ }^{1}$, Xiangjun Chen ${ }^{2,3,4,5,6}$, Neil S. Lagali ${ }^{7}$, Sten Ræder ${ }^{2}, \varnothing_{\text {ygunn A. Utheim }}^{8}$, Darlene A. Dartt ${ }^{9}$, Tor P. Utheim $^{4,5,6,7,10,11,12}$

${ }^{1}$ Institute of Clinical Medicine, Faculty of Medicine, University of Oslo, Oslo, Norway

${ }^{2}$ The Norwegian Dry Eye Clinic, Oslo, Norway

${ }^{3}$ Department of Ophthalmology, Arendal Hospital, Arendal, Norway

${ }^{4}$ Department of Ophthalmology, Vestre Viken Hospital Trust, Drammen, Norway

${ }^{5}$ Faculty of Health Sciences, National Centre for Optics, Vision and Eye Care,

University College of Southeast Norway, Norway

${ }^{6}$ Department of Oral Surgery and Oral Medicine, Faculty of Dentistry, Univeristy of Oslo, Norway

${ }^{7}$ Department of Clinical and Experimental Medicine, Linköping University, Linköping, Sweden.

${ }^{8}$ Department of Ophthalmology, Oslo University Hospital, Oslo, Norway

${ }^{9}$ Department of Ophthalmology, Harvard Medical School, Boston, Massachusetts, United States

${ }^{10}$ Department of Medical Biochemistry, Oslo University Hospital, Norway

${ }^{11}$ Department of Plastic and Reconstructive Surgery, Oslo University Hospital, Oslo, Norway

${ }^{12}$ Department of Maxillofacial Surgery, Oslo University Hospital, Norway

Financial Support: none

Conflict of Interest: no conflicting relationship exists for any author

Meeting Presentation: Parts of the material was presented at the Nordic Congress of Ophthalmology in Oslo, Norway on $22^{\text {nd }}-25^{\text {th }}$ August.

\section{Corresponding author:}

Muhammed Yasin Adil

E-mail: m_yasin_93@hotmail.com

Mobile: +47 41769179

Adress: Oekernveien 250, 0584 Oslo 


\begin{abstract}
Purpose: To investigate the relationship between meibomian gland (MG) morphology and clinical dry eye tests in patients with meibomian gland dysfunction (MGD).

Design: Cross-sectional study.

Subjects: Total 538 MGD patients, and 21 healthy controls.

Methods: MG loss on meibography images of upper (UL) and lower lids (LL) was graded on a scale of 0 (lowest degree of MG loss) to 3. MG length, thickness and inter-glandular space in the UL were measured. Clinical tests included meibum expression and quality, tear-film breakup time, ocular staining, osmolarity, Schirmer I, blink interval timing and Ocular Surface Disease Index (OSDI) questionnaire. Results: Mean UL and LL meibogrades were significantly higher in MGD patients compared to controls ( $\mathrm{P}<0.001$ for UL and LL). The sensitivity and specificity of the meibograde as a diagnostic parameter for MGD was $96.7 \%$ and $85 \%$, respectively. Schirmer I was significantly increased in MGD patients with meibograde 1 compared to patients with meibograde 0,2 and 3 in the UL $(\mathrm{P}<0.05)$. MG thickness increased with higher meibograde $(\mathrm{P}<0.001)$. MG morphology correlated significantly but weakly with several clinical parameters $(\mathrm{P}<0.05)$. OSDI did not correlate with any MG morphological parameter.

Conclusions: Grading of MG loss using meibograde effectively diagnoses MGD. Compensatory mechanisms such as increased aqueous tear production and dilatation of MGs make early detection of MGD difficult by standard clinical measures of dry eye, whereas morphologic analysis of MGs reveals an early stage of MGD, and therefore represent a complementary clinical parameter with diagnostic potential.
\end{abstract}




\section{Introduction}

Meibomian glands (MG) are responsible for the secretion of meibum, which constitutes the main lipid component of the outer layer of the tear-film. ${ }^{1}$ These lipids stabilize and prevent early evaporation of the tear-film, and are consequently critical to ensure a healthy ocular surface. Therefore, alterations in meibum, whether due to deficiencies in secretion or the composition of lipids, have adverse effects on the tearfilm, resulting in evaporative dry eye disease (DED). ${ }^{1-3}$ Meibomian gland dysfunction (MGD) is reported to be the most common cause of evaporative DED, and is a frequent condition encountered in ophthalmologic practice ${ }^{4-7}$ Epidemiological studies report the prevalence of MGD in different populations to range from $38 \%$ to $68 \% .^{8}$

Recent advances in non-invasive techniques of visualizing MG morphology provide clinicians with a novel tool in diagnosis and classification of MGD. ${ }^{9-11}$ Nevertheless, studies investigating the relationship between MG morphology and clinical tests associated with DED report inconsistent results. ${ }^{12-17}$ Previous reports also showed that certain morphologic characteristics such as MG thickness, length and tortuosity are associated with different stages of MGD. ${ }^{1,2,18}$ The nature of MGD, with its various presentations, has therefore resulted in a lack of clearly defined diagnostic criteria and an absence of effective diagnostic tools for MGD.

Loss of MG tissue is most commonly the sole investigated characteristic on meibography images, and the International Workshop on MGD recognizes MG loss as a key clinical sign of MGD. ${ }^{1,9,19-21}$ Studies have, however, shown a regional difference of actively secreting MGs on the tarsal plate, ${ }^{22}$ compensatory thickening in early stage MGD, ${ }^{18,23}$ and a low utilization of the meibum reservoir in healthy subjects. $^{24,25}$ Therefore, morphologic alterations in MGD should not be restricted to atrophy alone. Further analyses of additional parameters of MG morphology and function and their relation to clinical dry eye tests are required.

In this study, we aimed to evaluate MG morphology as a potential discriminator between MGD patients and healthy subjects, and to better understand the pathophysiological changes that occur with MGD development. Herein, we investigated the relationship between morphological characteristics of MGs visualized by meibography and clinical dry eye tests, in a large sample of a Norwegian cohort of patients with MGD. Additionally, we explored the differences in clinical and morphologic parameters in distinct MG loss groups, compared with age-matched healthy subjects.

\section{Methods}

A total of 538 patients seeking consultation at the Norwegian Dry Eye Clinic, and diagnosed with MGD according to the guidelines from 2011, ${ }^{1}$ were included in this cross-sectional study. Briefly, the diagnosis of MGD is made after first diagnosing evaporative DED, based upon symptoms assessment and tear-film break-up time (TFBUT), and supplementary clinical tests such as blink rate and interval timing, tearfilm osmolarity measurement, Schirmer I test and ocular staining. Additionally, MGD specific assessments of morphologic eyelid features, meibum expressibility and quality, and evaluation of gland dropout on meibography images are used to separate MGD from other subtypes of DED. All data were collected from the first consultation at the clinic. Twenty-one healthy volunteers, with no pre-existing ocular or systemic conditions or symptoms of DED, were recruited through the National Centre for Optics, Vision and Eye Care as a control group. Written informed consent was obtained from all participants prior to examination. The study was conducted in accordance with the Declaration of Helsinki. The Regional Committee for Medical \& 
Health Research Ethics, Section C, South East Norway (REC) reviewed the use of the data material from the clinic. REC found the research project "Evaluation of data from the Norwegian Dry Eye Clinic" to be outside the remit of the Act on Medical and Health Research (2008) and, therefore, be implemented without specific approval. A letter of exemption from REC has been provided.

\section{Meibography}

Meibography images were acquired by the non-contact infrared meibography system Oculus Keratograph 5 (Oculus, Wezlar, Germany). Images of both upper (UL) and lower (LL) eyelids of both eyes were analyzed. Subjects were excluded on the basis of unsatisfactory meibography images by the application of the following exclusion criteria; 1) interrupted complete assessment of eyelid, i.e. nail/finger; 2) inadequate exposure of the tarsal area; 3 ) interfering reflection; or 4) lack of focus/blurry image.

An experienced observer subjectively evaluated the MG loss in both UL and LL using a validated meibograde grading scheme, with a four-point scale from 0-3: where grade 0 : area of MG loss between 0-25\%; grade 1: area of MG loss between 26-50 \%; grade 2: area of MG loss between 51-75\%; and grade 3: area of MG loss $76-100 \%$ (Figure 1). The area of MG loss was evaluated in reference to expected normal MG area in healthy subjects, equivalent to the tarsal plate. ${ }^{10}$

Following the subjective evaluation, an extensive computerized objective assessment was performed with ImageJ (v. 2.0.0) software. Briefly, the tarsal area was outlined, as the assumed normal MG area, using ImageJ's polygon function. The outer boundaries of this area were identified according to the definitions proposed by Pult et al. ${ }^{19}$ To increase visibility of the MGs, local contrast was enhanced using a plug-in function in ImageJ. Finally, the actual MG area was outlined, and the percentage of MG loss was calculated by dividing the MG area by the tarsal area (Figure 2A and 2B).

Additional computerized analyses of MG morphology included measurements of length, thickness and inter-glandular space (i.e. the space between two adjacent MGs), which were performed on the three most representative glands in the UL only as it often was difficult to distinguish separate MGs in the LL for morphologic investigations (Figure 2C). Average values of these measurements were used for further statistical analysis. Furthermore, the number of tortuous MGs (tortuosity) was reported, with a tortuous gland being defined as having at least one angle greater than 45 degrees.

\section{Clinical Dry Eye Tests}

Patients first completed a symptom questionnaire to give an Ocular Surface Disease Index (OSDI) score between 0 (no symptoms) and 100 (severe symptoms). ${ }^{26}$ Meibum expression was measured by application of firm digital pressure to the central area of the LL to observe the number of active MGs. Five MGs in the central area were tested for their ability to express meibum. This result was scored from $0-3 ; 0=$ all glands expressible; 1 = 3-4 gland expressible; 2 = 1-2 glands expressible; and $3=$ no glands expressible. ${ }^{27}$ Meibum quality of the central eight MGs was scored from 0-3; $0=$ clear fluid; 1 = cloudy fluid; 2 = cloudy; particulate fluid and 3 = opaque, toothpaste-like meibum. ${ }^{28}$ The score for each gland was added to give a total score. MGs that were not able to express meibum were scored 0 for meibum quality with an additional note in the journal system. ${ }^{1}$

Ocular staining and TFBUT were measured 0.5 minutes after instillation of 5 $\mu 10.5 \%$ fluorescein to the conjunctival sac of each eye, and graded according to the 
Oxford grading system. ${ }^{1,29}$ Furthermore, to quantitatively assess tear-secretion, Schirmer I test was performed without anesthesia by inserting the test strip in the lateral third of the lower eyelid for 5 minutes. ${ }^{1}$

Additional clinical tests included timing of blink interval, measurement of tear-film osmolarity with the TearLab® TM system (TearLab Corporation, San Diego, CA), and evaluation of Dry Eye Severity Level (DESL) from 1-4 according to the guidelines proposed by the 2007 International Dry Eye Workshop. ${ }^{30}$

\section{Statistical Analysis}

Statistical analysis was performed using SPSS (v. 24.0) and GraphPad Prism (v.7.0) statistical software. Data was tested for normal distribution by Shapiro-Wilks test. Nonparametric tests were used; Spearman rank correlation coefficient was calculated for correlations, Mann-Whitney U statistics and Kruskall-Wallis with Dunn's posthoc test were performed to compare groups. For comparisons between the patient group and the control group, the influence of age was adjusted for using a general linear model (GLM). The significances of multiple correlations were corrected with the two-stage linear step-up procedure of Benjamini, Krieger and Yekutieli (q-value was set at 1\%). A Receiver Operator Characteristics (ROC) curve was generated to investigate the clinical application and cut-off values of the meibograde system for establishing MGD diagnosis. With the exception of ROC statistics, values from the right and left eyes were combined to give one value for UL and one for LL in all subjects. For ROC statistics, all four eyelids were averaged to give one meibograde for each subject. Clinical dry eye tests that were performed on each eye separately were combined to give a mean value. A p-value $<0.05$ was considered significant.

\section{Results}

Fifty-one patients and one control subject were excluded from the study prior to commencing analyses due to unsatisfactory meibography images according to the exclusion criteria. Thus, meibography images of 487 patients (361 female and 126 male; age: $50.13 \pm 16.37$ years, range: 9 to 88 years) and 20 healthy controls (12 female and 8 male; age: $31.7 \pm 14$ years, range: 19 to 65 years) were included, resulting in a total of 2028 images.

We found significantly higher values in mean meibograde, as well as percentage dropout by computerized assessment, for both UL and LL in MGD patients compared to that of healthy subjects. Mean UL and LL meibogrades were $1.15 \pm 1.09$ and $1.8 \pm 0.95$ respectively, in patients, while these values were $0.1 \pm 0.3$ and $0.25 \pm 0.49$ in the control group ( $\mathrm{P}<0.001$ for controls vs. patients, for UL and LL). The distribution of MGD patients with different meibogrades can be seen in table 1 . Using computerized analysis, the mean percentage MG loss in the UL was $31.3 \pm 17 \%$ in patients and $13.8 \pm 5.5 \%$ in controls, while dropout in the LL was $51.7 \pm 14.6 \%$ in patients and $23.6 \pm 13.2 \%$ in controls $(\mathrm{P}<0.001$ for controls vs. patients, for UL and LL). These data suggested increased loss of MG tissue in MGD patients compared to healthy subjects.

We generated a Receiver Operator Characteristics (ROC) curve and calculated the area-under-the-curve (AUC) to assess the ability of the subjective meibograde grading system to discriminate between MGD patients and healthy controls (Figure 3 ). The highest AUC, and consequently the optimal cut-off value, was achieved using an average of meibogrades for ULs and LLs in both the right and left eyes (average meibograde of all four eyelids). The average meibograde for patients was significantly higher than control values (mean $2.57 \pm 1.32$ and $0.35 \pm 0.37$, respectively, 
Mann-Whitney U, P < 0.001). The AUC was calculated to be $0.970(\mathrm{P}<0.001)$. For a cut-off value of average meibograde 0.5 , the sensitivity and specificity were $96.7 \%$ and $85 \%$, respectively. Using average meibograde 0.75 as a cut-off value resulted in a sensitivity of $87.9 \%$ and a specificity of $100 \%$. Furthermore, 481 out of 487 MGD patients had an average meibograde of at least 0.5 . These numbers demonstrate that the symptomatic MGD population in our study with average meibograde below 0.5 is quite sparse.

Schirmer I test was significantly higher in patients with meibograde 1 in the UL compared to patients with meibograde 0, 2 and 3 ( $\mathrm{P}<0.05)$ (Table 2A).

Additionally, the mean value of the Schirmer I test among all MGD patients was insignificant from the control group, $14.4 \pm 10 \mathrm{~mm}$ in patients and $16.5 \pm 11.9 \mathrm{~mm}$ in the control group respectively $(\mathrm{P}=0.40)$ (Table 3$)$.

MG thickness in the UL increased significantly with higher subjective meibograde in the MGD group (Kruskal-Wallis $\mathrm{P}<0.001$ ). Inter-glandular space increased with progressive MG loss despite increasing MG thickness, whereas MG length and tortuosity was extensively reduced with higher meibograde $(\mathrm{P}<0.001)$ (Table 4).

We found several changes in clinical dry eye tests, in both ULs and LLs with different meibogrades in the MGD group (Table 2A and 2B). Ocular staining, DESL and meibum expression were increased in patients with higher meibogrades, whereas changes in OSDI, TFBUT and meibum quality in the UL were significant, but subtle, and did not follow any particular pattern. Osmolarity was significantly increased in MGD patients in comparison to controls (Table 3).

Amongst MGD patients, meibum expression correlated significantly with percentage MG loss (by computerized assessment) in the UL $(\mathrm{r}=0.368, \mathrm{P}<0.001)$ and LL $(r=0.202, P<0.001)$, and inversely to MG length $(r=-0.286, P<0.001)$. MG thickness was inversely correlated to osmolarity $(\mathrm{r}=-0.198, \mathrm{P}<0.001)$. Weak but significant correlations were found for several parameters (Table 5). OSDI did not correlate with any parameter of MG morphology. Age was not correlated with either UL meibograde $(r=0.055, P=0.085)$ or LL meibograde $(r=0.018, P=0.582)$, in the MGD group. Similarly, in the control group, age was not correlated with UL meibograde $(\mathrm{r}=-0.294, \mathrm{P}=0.066)$ or LL meibograde $(\mathrm{r}=-0.025, \mathrm{P}=0.879)$. The average meibograde for all four eyelids in a patient correlated with age $(\mathrm{r}=0.286, \mathrm{P}<$ $0.001)$ in MGD patients, but not in the control group $(\mathrm{r}=0.194, \mathrm{P}=0.413)$.

\section{Discussion}

In the present study, we investigated MG morphology assessed by meibography in relation to common clinical tests used to evaluate DED. We found significantly higher MG loss in MGD patients compared to controls. Our subjective meibograde grading system showed excellent ability to discriminate between MGD patients and controls. Furthermore, we observed increased tear-fluid production in early-phase MGD and thickening of MGs with increasing subjective meibograde scores in MGD patients. Moreover, clinical parameters were weakly correlated to MG morphology and symptoms score was not related to changes in MG morphology.

The varied presentation of MGD makes early detection of MGD development complicated. Therefore, an effective and reliable clinical screening test is strongly needed. Previous studies have evaluated grading of meibography as a reliable clinical test. $^{31,32}$ Furthermore, Arita et al. demonstrated the diagnostic ability of their meibography grading scheme to discriminate between patients and healthy subjects. ${ }^{14}$ A number of different grading scales have been used in previous studies, including 
four-point, ${ }^{20,33}$ five-point,,${ }^{31}$ and seven-point grading scales. ${ }^{34}$ All of these define the lowest grade (grade or score 0) as no MG loss or no gland dropout. A previous report suggested a cut-off value of $16.9 \%$ (upper eyelid) and $28.7 \%$ (lower eyelid) in MG loss for the purpose of discrimination between dry eye and normal eyes. ${ }^{19}$ This demonstrates high variability of mild MG loss within the healthy population. Using a grade defined as $0 \%$ when such high variability has been demonstrated could potentially create a bias and a weakness in the grading of MG loss. Therefore, we employed a modified four-point grading scale for MG loss. ${ }^{20}$ More specifically, we use even increments of quartiles, including grade 0 being defined up to $25 \%$ MG loss, in an attempt to account for normal variation within the healthy population. Our modified meibograde showed excellent ability to differentiate between MGD and healthy subjects, with high sensitivity and specificity, using an average meibograde value for all four eyelids. These results are useful to establish the clinical application of meibography imaging in early detection of MGD by evaluation of MG loss.

Schirmer I test indicate changes in tear fluid production that is diagnostic of aqueous-deficient dry eye. In the current study, in both patient and control groups, the mean value of Schirmer I test was well within normal range and above the lower cutoff values for DED $(<5.5 \mathrm{~mm})$. Neither the patients nor the controls appear to have aqueous-deficient dry eye. ${ }^{1}$ Furthermore, there were no significant differences between Schirmer I values in the patient and control group. Arita et al. reported higher Schirmer 1 values in MGD patients in comparison to healthy subjects, and proposed increased tear-fluid production as a compensatory response in MGD patients. ${ }^{35}$ However, we did not observe higher Schirmer I values in MGD patients compared to normal controls. Nevertheless, our study showed that Schirmer I was increased in patients with UL meibograde 1, compared to patients with meibogrades 0,2 or 3 . Thus, we suggest that a potential compensatory increase in tear-fluid production that occurs due to MG loss and tear-film lipid layer dysfunction, which may only appear in early stage MGD. Contrary to the findings by Arita et al., ${ }^{35}$ we noted a trend that may indicate a reduction in tear-fluid production in severe MGD. This is in line with studies reporting that MGD and aqueous-deficient DED may overlap and occur simultaneously in DED. ${ }^{36}$ A possible explanation is that either the MGs or the lacrimal glands are exhausted after compensatory attempts in advance stages of MGD, secondary to either MGD or aqueous-deficient DED. However, as this was only noted for the upper eyelid, and Schirmer I measures reflex tear-fluid production, confounding factors, such as inflammation, may contribute to this observation.

We found that MG thickness increased with progressive MG loss. Several studies previously hypothesized that MG thickness is observed as a compensatory or secondary response. ${ }^{18,23,37}$ Studies also reported dilation of the secretory acini in MGs, indicating a cellular response to MG loss and reduced meibum secretion or increased meibum demand. ${ }^{37,38}$ The mechanisms underlying the dilatation of the secretory acini in MGs are unknown. Compensatory cellular repair mechanisms could result in hyperplastic acini with increased lipid production. ${ }^{18,39}$ Whereas the increase in thickness and dilation of secretory acini may increase meibum production and secretion, this process could be exhausted over time. Accordingly, we found that despite the increasing MG thickness, MG expressibility gradually worsened with higher meibograde. Interestingly, we also observed that the inter-glandular space increased in patients with higher meibogrades, especially those with meibograde 3 . Thus, despite the increasing thickness of single MGs, the space between MGs also increased. An explanation could be that MGD patients could have atrophy of entire 
glands. ${ }^{18}$ This would allow for the remaining MGs to thicken and the inter-glandular space to increase, indicating progressive total atrophy of the MG tissue. Together, these findings demonstrate that MG thickening is an inadequate response to progressive glandular atrophy.

A previous report revealed that MG loss is directly linked to reduction of the lipid layer thickness in the tear-film, ${ }^{40}$ which leads to shorter TFBUT. Thinner lipid layer thickness is associated with increased friction during blinking. ${ }^{41} \mathrm{We}$ observed reduced TFBUT in our patient group, and also significantly shorter blink intervals in comparison to the healthy subjects. Blinking rate was suggested as a compensatory as compensatory measure in DED, as blinking aids in distribution of tear-fluid across the ocular surface. ${ }^{42,43}$ Furthermore, hyperkeratinization of MG orifices is thought of as a major contributor of MGD pathogenesis. The obstruction of the gland orifice would lead to an increase in intraductal pressure and consequently dilatation of MGs. More frequent blinking and increased friction can worsen the increase in intraductal pressure caused by gland obstruction. ${ }^{2,18}$ Thus, we speculate that the increase in MG thickness could be a consequence of the increasing intraductal pressure rather than the lack of meibum secretion, ${ }^{2,18,44}$ making it a secondary, non-functional change. On the other hand, in the present study we demonstrated that MG thickness was inversely correlated to tear-film osmolarity and meibum expression, which may indicate a functional compensatory aspect to the increase in MG thickness. Taken together, our findings may support a combined explanation for increased MG thickness, which can be attributed to a compensatory response due to increased meibum demand and/or dilatation caused by increased intraductal pressure.

The tear-film osmolarity tends to be elevated in MGD patients. ${ }^{45-48}$ However weak, this relationship may point to a functional increase in MG thickness that could help to maintain osmolarity levels. Arita et al. also proposed that increased tear fluid production, which we found in our study in early-phase MGD, might contribute to preventing elevated osmolarity levels. ${ }^{35}$ Additionally, the increase in MG thickness appears to have a weak positive effect on meibum expression. Thus, the increase in MG thickness, together with the increase in tear-fluid production, may play partial roles in attempting to maintain ocular surface homeostasis with the onset of MGD.

Most clinical dry eye tests demonstrated subtle changes with increasing meibograde. OSDI scores were also independent of MG loss in the patient group. Previous studies have shown that the meibum reservoir in humans is much greater than the amount of meibum actually used in the tear-film lipid layer. ${ }^{24,25}$ This may partly explain why the tear meniscus height in MGD patients is comparable to that of healthy subjects. ${ }^{41}$ The lack of major changes in clinical dry eye test results and symptoms with increasing meibograde could therefore be due to the unutilized meibum reservoir in the patients included in our study. However, the patients in this study have test results beyond cut-offs for MGD. Thus, it is likely that this reservoir has already been depleted. Further development of MGD, could at this point be slow in nature. On this basis, we propose that there is a threshold for MGD, with regard to MG loss, after which symptoms occur, which also explains the existence of an asymptomatic MGD population., 7, 49, 50

In this study, we observed that MG morphology was only weakly correlated to clinical dry eye tests. Contrary to our findings, Pult et al. reported significantly stronger correlation coefficients. ${ }^{12,19}$ Most studies have, however, reported inconsistent results with regard to the relationship between MG loss and clinical tests. $^{11,13-17,21,22}$ Consistent with our findings, these studies also show that the strongest relationships were seen between MG morphology and meibum expression. 
Interestingly, we found no correlations between meibum quality and MG morphology. It may be noted, however, that meibum expression only indicates whether a gland is able to secrete meibum, and not the amount of meibum secreted. Furthermore, in this study, the quality of meibum is only detected grossly by eye, and the actual lipid composition of the meibum remains unexplored. Changes in MG morphology may not directly affect the ability of a gland to secrete, or the macroscopic quality of the meibum. It could, however, have an impact on the amount of meibum secreted and/or the microscopic composition of the lipid layer.

Furthermore, as inexpressible glands are given a score of 0 for meibum quality, this may contribute to a bias in the meibum quality scores among patients with low meibum expressibility scores.

In conclusion, MG loss appears to be the hallmark of MGD development, and the subjective meibograde score was an effective diagnostic tool for MGD. Our meibograde demonstrated excellent ability to differentiate MGD patients and healthy subjects, and could be utilized in clinical investigation and screening of early stage MGD. Changes in MG morphology were only weakly associated with clinical signs and dry eye test values, and were not linked to symptom score. Development of MGD could be attenuated by compensatory mechanisms, such as increased aqueous tearfluid production and increased thickness of MGs, making early detection of MGD difficult by standard clinical measures of dry eye, but possible using morphologic MG analysis. Investigations of MG morphology visualized by meibography therefore represent a complementary clinical parameter with diagnostic potential.

\section{Acknowledgments}

Funding/Support: none

Financial Disclosures: No financial disclosures. 


\section{References}

1. Tomlinson A, Bron AJ, Korb DR, et al. The international workshop on meibomian gland dysfunction: report of the diagnosis subcommittee. Invest Ophthalmol Vis Sci 2011;52(4):2006-49.

2. Baudouin C, Messmer EM, Aragona P, et al. Revisiting the vicious circle of dry eye disease: a focus on the pathophysiology of meibomian gland dysfunction. Br J Ophthalmol 2016;100(3):300-6.

3. McCulley JP, Shine WE. Meibomian gland function and the tear lipid layer. Ocul Surf 2003;1(3):97-106.

4. Schaumberg DA, Nichols JJ, Papas EB, Tong L, Uchino M, Nichols KK. The international workshop on meibomian gland dysfunction: report of the subcommittee on the epidemiology of, and associated risk factors for, MGD. Invest Ophthalmol Vis Sci 2011;52(4):1994-2005.

5. Foulks GN, Nichols KK, Bron AJ, Holland EJ, McDonald MB, Nelson JD. Improving awareness, identification, and management of meibomian gland dysfunction. Ophthalmology 2012;119(10 Suppl):S1-12.

6. Lemp MA, Nichols KK. Blepharitis in the United States 2009: a surveybased perspective on prevalence and treatment. Ocul Surf 2009;7(2 Suppl):S1s14.

7. Lemp MA, Crews LA, Bron AJ, Foulks GN, Sullivan BD. Distribution of aqueous-deficient and evaporative dry eye in a clinic-based patient cohort: a retrospective study. Cornea 2012;31(5):472-8.

8. Stapleton F, Alves M, Bunya VY, et al. TFOS DEWS II Epidemiology Report. Ocul Surf 2017;15(3):334-365.

9. Wise RJ, Sobel RK, Allen RC. Meibography: A review of techniques and technologies. Saudi J Ophthalmol 2012;26(4):349-56.

10. Arita R, Itoh K, Maeda S, Maeda K, Amano S. A newly developed noninvasive and mobile pen-shaped meibography system. Cornea 2013;32(3):242-7.

11. Foulks GN, Bron AJ. Meibomian gland dysfunction: a clinical scheme for description, diagnosis, classification, and grading. Ocul Surf 2003;1(3):107-26.

12. Pult H, Riede-Pult BH. Non-contact meibography: keep it simple but effective. Cont Lens Anterior Eye 2012;35(2):77-80.

13. Feng Y, Gao Z, Feng K, Qu H, Hong J. Meibomian Gland Dropout in Patients with Dry Eye Disease in China. Current Eye Research 2014;39(10):965-972.

14. Arita R, Itoh K, Maeda S, et al. Proposed diagnostic criteria for obstructive meibomian gland dysfunction. Ophthalmology 2009;116(11):2058-63.e1.

15. Cuevas M, Gonzalez-Garcia MJ, Castellanos E, et al. Correlations among symptoms, signs, and clinical tests in evaporative-type dry eye disease caused by Meibomian gland dysfunction (MGD). Curr Eye Res 2012;37(10):855-63.

16. Jie $\mathrm{Y}, \mathrm{Xu} \mathrm{L}, \mathrm{Wu} \mathrm{YY}$, Jonas JB. Prevalence of dry eye among adult Chinese in the Beijing Eye Study. Eye (Lond) 2009;23(3):688-93.

17. Srinivasan S, Menzies K, Sorbara L, Jones L. Infrared imaging of meibomian gland structure using a novel keratograph. Optom Vis Sci 2012;89(5):788-94.

18. Knop E, Knop N, Millar T, Obata H, Sullivan DA. The international workshop on meibomian gland dysfunction: report of the subcommittee on 
anatomy, physiology, and pathophysiology of the meibomian gland. Invest Ophthalmol Vis Sci 2011;52(4):1938-78.

19. Pult H, Riede-Pult BH, Nichols JJ. Relation between upper and lower lids' meibomian gland morphology, tear film, and dry eye. Optom Vis Sci 2012;89(3):E310-5.

20. Arita R, Itoh K, Inoue K, Amano S. Noncontact infrared meibography to document age-related changes of the meibomian glands in a normal population. Ophthalmology 2008;115(5):911-5.

21. Finis D, Ackermann P, Pischel N, et al. Evaluation of Meibomian Gland Dysfunction and Local Distribution of Meibomian Gland Atrophy by Non-contact Infrared Meibography. Curr Eye Res 2015;40(10):982-9.

22. Korb DR, Blackie CA. Meibomian gland diagnostic expressibility: correlation with dry eye symptoms and gland location. Cornea 2008;27(10):1142-7.

23. Korb DR, Henriquez AS. Meibomian gland dysfunction and contact lens intolerance. J Am Optom Assoc 1980;51(3):243-51.

24. Chew CK, Hykin PG, Jansweijer C, Dikstein S, Tiffany JM, Bron AJ. The casual level of meibomian lipids in humans. Curr Eye Res 1993;12(3):255-9.

25. Blackie CA, Korb DR. The diurnal secretory characteristics of individual meibomian glands. Cornea 2010;29(1):34-8.

26. Schiffman RM, Christianson MD, Jacobsen G, Hirsch JD, Reis BL. Reliability and validity of the Ocular Surface Disease Index. Arch Ophthalmol 2000;118(5):615-21.

27. Pflugfelder SC, Tseng SC, Sanabria O, et al. Evaluation of subjective assessments and objective diagnostic tests for diagnosing tear-film disorders known to cause ocular irritation. Cornea 1998;17(1):38-56.

28. Bron AJ, Benjamin L, Snibson GR. Meibomian gland disease. Classification and grading of lid changes. Eye (Lond) 1991;5 ( Pt 4)(395-411.

29. Bron AJ, Evans VE, Smith JA. Grading of corneal and conjunctival staining in the context of other dry eye tests. Cornea 2003;22(7):640-50.

30. The definition and classification of dry eye disease: report of the Definition and Classification Subcommittee of the International Dry Eye WorkShop (2007). Ocul Surf 2007;5(2):75-92.

31. Pult H, Riede-Pult B. Comparison of subjective grading and objective assessment in meibography. Cont Lens Anterior Eye 2013;36(1):22-7.

32. Dogan AS, Kosker M, Arslan N, Gurdal C. Interexaminer Reliability of Meibography: Upper or Lower Eyelid? Eye Contact Lens 2016.

33. Nichols JJ, Berntsen DA, Mitchell GL, Nichols KK. An assessment of grading scales for meibography images. Cornea 2005;24(4):382-8.

34. Ngo W, Srinivasan S, Schulze M, Jones L. Repeatability of grading meibomian gland dropout using two infrared systems. Optom Vis Sci 2014;91(6):658-67.

35. Arita R, Morishige N, Koh S, et al. Increased Tear Fluid Production as a Compensatory Response to Meibomian Gland Loss: A Multicenter Cross-sectional Study. Ophthalmology 2015;122(5):925-33.

36. Nichols KK, Foulks GN, Bron AJ, et al. The international workshop on meibomian gland dysfunction: executive summary. Invest Ophthalmol Vis Sci 2011;52(4):1922-9. 
37. Knop E, Knop N. [Meibomian glands : part IV. Functional interactions in the pathogenesis of meibomian gland dysfunction (MGD)]. Ophthalmologe 2009;106(11):980-7.

38. Straatsma BR. Cystic degeneration of the meibomian glands. AMA Arch Ophthalmol 1959;61(6):918-27.

39. Horsley V, O'Carroll D, Tooze R, et al. Blimp1 defines a progenitor population that governs cellular input to the sebaceous gland. Cell 2006;126(3):597-609.

40. Eom Y, Lee JS, Kang SY, Kim HM, Song JS. Correlation between quantitative measurements of tear film lipid layer thickness and meibomian gland loss in patients with obstructive meibomian gland dysfunction and normal controls. Am J Ophthalmol 2013;155(6):1104-1110.e2.

41. Tung CI, Perin AF, Gumus K, Pflugfelder SC. Tear meniscus dimensions in tear dysfunction and their correlation with clinical parameters. Am J Ophthalmol 2014;157(2):301-310.e1.

42. Tsubota K, Hata S, Okusawa Y, Egami F, Ohtsuki T, Nakamori K. Quantitative videographic analysis of blinking in normal subjects and patients with dry eye. Arch Ophthalmol 1996;114(6):715-20.

43. Korb DR, Baron DF, Herman JP, et al. Tear film lipid layer thickness as a function of blinking. Cornea 1994;13(4):354-9.

44. Bron AJ, Tiffany JM. The contribution of meibomian disease to dry eye. Ocul Surf 2004;2(2):149-65.

45. Mathers WD, Shields WJ, Sachdev MS, Petroll WM, Jester JV. Meibomian gland dysfunction in chronic blepharitis. Cornea 1991;10(4):277-85.

46. Mathers WD, Lane JA, Sutphin JE, Zimmerman MB. Model for ocular tear film function. Cornea 1996;15(2):110-9.

47. Gilbard JP, Rossi SR, Heyda KG. Tear film and ocular surface changes after closure of the meibomian gland orifices in the rabbit. Ophthalmology 1989;96(8):1180-6.

48. Baudouin C, Aragona P, Messmer EM, et al. Role of hyperosmolarity in the pathogenesis and management of dry eye disease: proceedings of the OCEAN group meeting. Ocul Surf 2013;11(4):246-58.

49. Viso E, Rodriguez-Ares MT, Abelenda D, Oubina B, Gude F. Prevalence of asymptomatic and symptomatic meibomian gland dysfunction in the general population of Spain. Invest Ophthalmol Vis Sci 2012;53(6):2601-6.

50. Amano S, Inoue K. Estimation of Prevalence of Meibomian Gland Dysfunction in Japan. Cornea 2017;36(6):684-688. 


\section{Figure Captions}

\section{Figure 1 The Meibograde Grading System}

The meibograde grading system; subjective grading of meibomian gland loss.

Figure 2 Computerized Analyses of Meibomian Gland Morphology with ImageJ

Computerized grading of meibomian gland loss in the upper eyelid (A) and lower eyelid (B) using ImageJ software. The contrast is enhanced on the tarsal area to improve visualization of the meibomian glands. Image $\mathrm{C}$ show computerized semiobjective measurements of meibomian gland length (blue), thickness (red), interglandular space (green) and tortuosity (yellow).

\section{Figure 3 The Diagnostic Ability of the Meibograde Grading System}

ROC-curve demonstrating the diagnostic ability of meibogrades for diagnosing MGD. Our results demonstrated excellent ability of the subjective meibograde to distinguish between MGD patients and healthy controls. Using an average meibograde of all four eyelids in a subjects, a cut-off value of average meibograde 0.5 for MGD resulted in a sensitivity and specificity of $96.7 \%$ and $85 \%$, respectively. 
Table 1 Age and number of meibomian gland dysfunction patients with different meibogrades in the upper and lower eyelid. The sub-groups of meibomian gland dysfunction patients with different meibogrades are age-matched.

\begin{tabular}{lccccc}
\hline Meibograde & $\mathbf{0}$ & $\mathbf{1}$ & $\mathbf{2}$ & $\mathbf{3}$ & P-value \\
\hline UL & $\begin{array}{c}49.2 \pm 16 \\
\text { years } \\
(\mathrm{n}=352\end{array}$ & $\begin{array}{c}50.1 \pm 16.6 \\
(\mathrm{n}=275)\end{array}$ & $\begin{array}{c}51 \pm 17.2 \\
(\mathrm{n}=185)\end{array}$ & $\begin{array}{c}51.4 \pm 15.9 \\
(\mathrm{n}=158)\end{array}$ & 0.758 \\
Leelids $)$ & & & & \\
LL & $\begin{array}{c}50.2 \pm 17.1 \\
(\mathrm{n}=101)\end{array}$ & $\begin{array}{c}50 \pm 15.7 \\
(\mathrm{n}=250)\end{array}$ & $\begin{array}{c}49.6 \pm 16.4 \\
(\mathrm{n}=363)\end{array}$ & $\begin{array}{c}51 \pm 16.7 \\
(\mathrm{n}=256)\end{array}$ & 0.441 \\
\hline UL: Upper Lid & & & & &
\end{tabular}

UL: Upper Lid, LL: Lower Lid 
Table 2A Comparison of clinical test results between meibomian gland dysfunction patients with different meibogrades in the upper eyelid.

\begin{tabular}{lccccc}
\hline $\begin{array}{l}\text { UL } \\
\text { meibograde }\end{array}$ & $\mathbf{0}$ & $\mathbf{1}$ & $\mathbf{2}$ & $\mathbf{3}$ & $\begin{array}{c}\text { Kruskall- } \\
\text { Wallis P- } \\
\text { value }\end{array}$ \\
\hline $\begin{array}{l}\text { OSDI } \\
\text { DESL }\end{array}$ & $33.6 \pm 22.7$ & $37.6 \pm 23.7$ & $38.8 \pm 20.3$ & $33 \pm 21.6$ & $<0.05$ \\
$\begin{array}{l}\text { Osmolarity } \\
\text { (mOsmol/L) }\end{array}$ & $311.8 \pm 0.3$ & $2.1 \pm 0.3$ & $2.1 \pm 0.4$ & $\mathbf{2 . 2} \pm \mathbf{0 . 4}$ & $<0.001$ \\
TFBUT (s) & $4.2 \pm 3$ & $312.5 \pm 15.9$ & $313.1 \pm 17.2$ & $310.4 \pm 14.4$ & 0.793 \\
$\begin{array}{l}\text { Blink } \\
\text { interval (s) }\end{array}$ & $3.3 \pm 2.7$ & $3.7 \pm 4.4$ & $\mathbf{3 . 2} \pm \mathbf{2 . 4}$ & $3.3 \pm 2.1$ & 0.001 \\
$\begin{array}{l}\text { Schirmer I } \\
\text { (mm) }\end{array}$ & $14.7 \pm 9.6$ & $\mathbf{1 6 . 9} \pm \mathbf{9 . 3}$ & $14.2 \pm 9.5$ & $14.2 \pm 9.4$ & 0.001 \\
$\begin{array}{l}\text { Ocular } \\
\text { Staining }\end{array}$ & $1.4 \pm 1.8$ & $1.6 \pm 2.2$ & $1.6 \pm 2$ & $\mathbf{1 . 9} \pm \mathbf{2 . 1}$ & 0.004 \\
$\begin{array}{l}\text { Meibum } \\
\text { expression }\end{array}$ & $0.9 \pm 0.9$ & $\mathbf{1 . 2} \pm \mathbf{0 . 9}$ & $\mathbf{1 . 4} \pm \mathbf{0 . 9}$ & $\mathbf{1 . 7} \pm \mathbf{0 . 9}$ & $<0.001$ \\
$\begin{array}{l}\text { Meibum } \\
\text { quality }\end{array}$ & $9.4 \pm 4.5$ & $10.3 \pm 4.5$ & $8.6 \pm 4.8$ & $9.2 \pm 6$ & 0.003 \\
$\begin{array}{l}\text { Values in bold are significant compared to Meibograde } 0 . \text { UL: Upper Lid, LL: Lower Lid, OSDI: Ocular Surface } \\
\text { Disease Index, DESL: Dry Eye Severity Level, TFBUT: Tear-Film Break-up Time. }\end{array}$ &
\end{tabular}


Table 2B Comparison of clinical test results between meibomian gland dysfunction patients with different meibogrades in the lower eyelid.

\begin{tabular}{|c|c|c|c|c|c|}
\hline $\begin{array}{l}\mathbf{L L} \\
\text { meibograde }\end{array}$ & $\mathbf{0}$ & 1 & 2 & 3 & $\begin{array}{c}\text { Kruskall- } \\
\text { Wallis P- } \\
\text { value } \\
\end{array}$ \\
\hline OSDI & $40.3 \pm 23.4$ & $36.2 \pm 22.5$ & $33.9 \pm 22.2$ & $35.9 \pm 22.6$ & 0.133 \\
\hline DESL & $2 \pm 0.2$ & $2.1 \pm 0.3$ & $2.1 \pm 0.3$ & $2.2 \pm 0.4$ & 0.004 \\
\hline $\begin{array}{l}\text { Osmolarity } \\
(\mathrm{mOsmol} / \mathrm{L})\end{array}$ & $311.9 \pm 13.9$ & $312.4 \pm 16.2$ & $312.5 \pm 15$ & $311 \pm 15.2$ & 0.8 \\
\hline TFBUT (s) & $3.8 \pm 2.6$ & $3.7 \pm 2.7$ & $3.8 \pm 2.8$ & $3.9 \pm 2.7$ & 0.678 \\
\hline $\begin{array}{l}\text { Blink } \\
\text { interval (s) }\end{array}$ & $3.2 \pm 2.4$ & $3.1 \pm 2.1$ & $3.8 \pm 5.3$ & $3.2 \pm 2.3$ & 0.183 \\
\hline $\begin{array}{l}\text { Schirmer I } \\
(\mathrm{mm})\end{array}$ & $15.6 \pm 10.1$ & $14.4 \pm 9.5$ & $15.2 \pm 9.8$ & $14.3 \pm 9.2$ & 0.568 \\
\hline $\begin{array}{l}\text { Ocular } \\
\text { Staining }\end{array}$ & $1 \pm 1.2$ & $1.5 \pm 2$ & $1.7 \pm 2.1$ & $1.7 \pm 2$ & 0.022 \\
\hline $\begin{array}{l}\text { Meibum } \\
\text { expression }\end{array}$ & $1.1 \pm 0.9$ & $1.1 \pm 0.9$ & $1.1 \pm 0.9$ & $1.5 \pm 1$ & $<0.001$ \\
\hline $\begin{array}{l}\text { Meibum } \\
\text { quality }\end{array}$ & $9.1 \pm 4$ & $10 \pm 4.6$ & $9.3 \pm 4.4$ & $9.3 \pm 5.8$ & 0.263 \\
\hline
\end{tabular}


Table 3 Comparison of clinical dry eye test results between meibomian gland dysfunction patients and healthy controls.

\begin{tabular}{lccc}
\hline & Patients & Controls & $\begin{array}{c}\text { Mann-Whitney U } \\
\text { P-value }\end{array}$ \\
\hline $\begin{array}{l}\text { Osmolarity } \\
\text { (mOsmol/L) }\end{array}$ & $311.9 \pm 14.4$ & $305 \pm 10.5$ & 0.006 \\
TFBUT $(\mathrm{s})$ & $3.7 \pm 2.7$ & $9.6 \pm 10$ & $<\mathbf{0 . 0 0 1}$ \\
Blink interval (s) & $3.4 \pm 4.1$ & $4.3 \pm 3.9$ & $\mathbf{0 . 0 1}$ \\
Schirmer 1 (mm) & $14.4 \pm 10$ & $16.5 \pm 11.9$ & 0.4 \\
Ocular Staining & $1.6 \pm 2$ & $1 \pm 0.7$ & 0.673 \\
Meibum expression & $1.2 \pm 0.9$ & $0.8 \pm 0.8$ & 0.002 \\
Meibum quality & $9.3 \pm 4.9$ & $4 \pm 0.2$ & $\mathbf{0 . 0 0 1}$ \\
\hline
\end{tabular}

Bold P-values indicate significance after adjusting for the influence of age using a General Linear Model. TFBUT: Tear-Film Break-up Time 
Table 4 Comparison of morphological parameters between meibomian gland dysfunction patients with different meibogrades in the upper eyelid.

\begin{tabular}{lccccc}
\hline $\begin{array}{l}\text { UL } \\
\text { Meibograde }\end{array}$ & $\mathbf{0}$ & $\mathbf{1}$ & $\mathbf{2}$ & $\mathbf{3}$ & $\begin{array}{c}\text { Kruskall- } \\
\text { Wallis P- } \\
\text { value }\end{array}$ \\
\hline Thickness & $18.8 \pm 3.3$ & $19.4 \pm 3.6$ & $\mathbf{1 9 . 9} \pm \mathbf{3 . 7}$ & $\mathbf{2 0 . 6} \pm \mathbf{4 . 5}$ & $<0.001$ \\
$\begin{array}{l}\text { Inter- } \\
\text { glandular }\end{array}$ & $14.9 \pm 2.7$ & $15.4 \pm 3$ & $15.5 \pm 3.2$ & $\mathbf{1 6 . 8} \pm \mathbf{3 . 6}$ & $<0.001$ \\
space & & & & & \\
$\begin{array}{l}\text { Length } \\
\text { Tortuosity }\end{array}$ & $308.2 \pm 53.4$ & $\mathbf{2 8 8 . 5} \pm \mathbf{5 2 . 5}$ & $\mathbf{2 1 9 . 5} \pm \mathbf{5 0 . 2}$ & $\mathbf{1 4 7} \pm \mathbf{5 3 . 2}$ & $<0.001$ \\
\hline
\end{tabular}

Values in bold are significant compared to Meibograde 0. Thickness, inter-glandular space, length and tortuosity all refer to meibomian glands. Values are given in ImageJ pixels. 
Table 5 Correlation coefficients of clinical dry eye tests and mebomian gland morphology in meibomian gland dysfunction patients.

\begin{tabular}{lccccccccc}
\hline & OSDI & DESL & TFBUT & $\begin{array}{c}\text { Osmo- } \\
\text { larity }\end{array}$ & $\begin{array}{c}\text { Blink } \\
\text { interval }\end{array}$ & $\begin{array}{c}\text { Schirmer } \\
\text { I }\end{array}$ & $\begin{array}{c}\text { Ocular } \\
\text { staining }\end{array}$ & $\begin{array}{c}\text { Meibum } \\
\text { expression }\end{array}$ & $\begin{array}{c}\text { Meibum } \\
\text { quality }\end{array}$ \\
\hline UL meibograde & .031 & $\mathbf{. 1 5 1}$ & $\mathbf{- . 1 2 2}$ & -.025 & $\mathbf{- . 0 6 9}$ & -.025 & $\mathbf{. 1 0 0}$ &. $\mathbf{2 9 0}$ & -.064 \\
LL meibograde & -.045 & $\mathbf{. 1 1 0}$ & .031 & -.023 & .020 & -.011 & $\mathbf{. 0 9 0}$ & $\mathbf{. 1 3 2}$ & -.048 \\
UL \% MG loss & .016 & $\mathbf{. 1 5 1}$ & -.095 & .011 & -.079 & -.031 & $\mathbf{. 1 1 0}$ &. $\mathbf{3 6 8}$ & -.075 \\
LL \% MG loss & -.038 & $\mathbf{. 1 5 1}$ & .037 & .017 & .034 & .016 & $\mathbf{. 1 3 5}$ &. $\mathbf{2 0 2}$ & -.078 \\
Thickness & -.006 & .006 & -.027 & -.196 & -.043 & -.057 & -.016 & $\mathbf{- 1 2 9}$ & .002 \\
Inter-glandular & -.019 & .027 & -.058 & $\mathbf{- 1 3 8}$ & -.045 & .014 & .011 & $\mathbf{- . 1 0 4}$ & .043 \\
space & & & & & & & & & \\
Length & -.010 & $\mathbf{- . 1 4 2}$ & .084 & -.040 & $\mathbf{. 0 9 9}$ & .042 & -.126 & -.286 & .076 \\
Tortuosity & -.065 & -.056 & .017 & -.020 & .077 & .044 & -.039 & $\mathbf{- . 1 0 7}$ & .049 \\
\hline
\end{tabular}

Significant values are highlighted in bold and were corrected for false discoveries using the procedure of Benjamini, Krieger and Yekutieli (q-value was set at 1\%). UL: Upper Lid, LL: Lower Lid, MG: Meibomian Gland, OSDI: Ocular Surface Disease Index, DESL: Dry Eye Severity Level, TFBUT: Tear-Film Break-up Time. 